\title{
Identidad, autonomía y concepciones de la buena vida
}

\author{
OSVALDO GUARIGLIA \\ Universidad de Buenos Aires (CONICET)
}

La modemidad establece las condiciones para un nucvo tipo de sujeto: aquel que se determina a sí mismo y que debe buscar su propia identidad en su historia y en la vida compartida con otros sujetos autónomos. Para cllo es central la concepción de autonomía que sostengamos. A fin de diluir oposiciones mal fundadas, en el trabajo se establece una diferencia conceptual entre una autonomía a), postulada, y una autonomia $b$ ), realizada. En cl otro extremo se analiza el concepto de autenticidad, que se ha solido contrastar con el de autonomía como un ideal alternativo de autorrealización. Se intenta demostrar que tanto el ideal filosófico de autonomía $b$ ) como el ideal post-romántico de autenticidad dan ambos por supuesta la vigencia de la autonomía a) como soporte y al mismo tiempo carácter distintivo de la identidad del sujeto moderno.

Desde el advenimiento de la modernidad existe una concepción del sujeto autónomo como un ser que se determina a sí mismo, en primer lugar asumiendo su propia existencia fáctica como una existencia limitada que tiene que vivirse, y luego como un Si mismo que debe buscar y hallar su propia identidad en su historia y en la vida compartida con otros sujetos. He desarrollado los aspectos más característicos de esta constitución del sujeto móral en el libro Moralidad ${ }^{1}$, de modo que es innecesario repetir aquí lo ya expresado en esa obra. La cuestión reside, sin embargo, en otro punto, estrechamente conectado con la génesis y la constitución de este sujeto moderno. En efecto, a partir de la coincidencia sobre las propiedades que lo determinan por oposición a los micmbros de sociedades tradicionales o jerárquicas, se han desarrollado dos corrientes distintas de interpretación del sujeto práctico, que a grandes rasgos se pueden identificar así: la primera, que pone el acento on el eje diacrónico de la formación del sujeto moral, está centrada básicamente en la hermenéutica contemporánea que pasa por la concepción de la autobiografía en W. Dilthey, el análisis existencial de Heidegger, el método interpretativo de Gadamer y desemboca en la actualidad en la concepción de la unidad narrativa del sujeto, desarrolladas paralelamente por Ch. Taylor ${ }^{2}$

\footnotetext{
'Guariglia, 1996, cap. 9, \& 3, pp. 233 y ss.

2 Taylor, 1989.
} 
y P. Ricoeur ${ }^{3}$; la segunda, que pone el acento en el eje sincrónico de la constitución del sujeto moral, estuvo formada originalmente por los teóricos fundadores de la sociología, E. Durkheim y G. H. Mead; del psicoanálisis, S. Freud, y de la psicología cognitiva, J. Piaget, y alcanza en la obra de L. Kohlberg ${ }^{4}$, J. Habermas ${ }^{5}$ y E. Tugendhat ${ }^{6}$ su concepción más acabada. Esta segunda corriente, a diferencia de la primera, sostiene la prioridad de la formación de una conciencia moral autónoma como la capacidad de comprender, elaborar y solucionar los conflictos morales de acuerdo con reglas generales compartidas por todos. En el libro citado más arriba, me he adherido a esta misma concepción del sujcto moral, sobre uno de cuyos aspectos desearía volver con mayor detalle. En efecto, yo sostengo contra los diversos comunitarismos que sólo una ética universalista puede ofrecer los soportes teóricos básicos de un sujeto práctico que integre en sí mismo la capacidad de deliberar y de elegir su propio ideal de buena vida, por una parte, y la capacidad de juzgar desde un punto de vista imparcial, en tercera persona, los actos morales propios y ajenos de acuerdo con los principios sustantivos universales de justicia, por la otra. A la inversa, me propongo ahora defender la tesis de que solamente a partir de la prioridad que concedamos a la autonomía sobre cualquier otra concepción de la buena vida, es posible explicar la unidad o identidad propia del sujeto moral a través de las múltiples vicisitudes que atraviesa en su vida. En otros términos, la unidad narrativa de una vida, como apropiadamente la ha denominado Ricoeur siguiendo a MacIntyre, es la que garantiza la identidad en el sentido de ipseidad del sujeto moral y está ligada de modo indisoluble a la concepción del propio proyecto de una buena vida ${ }^{7}$. A mi juicio, ese núcleo que constituye la ipseidad por encima y a contrapelo de todas las variaciones y profundos cambios a los que está sujeta una vida, puede sostenerse en carácter de tal sólo si se presupone la formación e integración en el sujeto moderno de una conciencia moral autónoma, la que, como tal, es impensable sin un sistema deóntico de normas y principios universales válidos y vigentes en la sociedad como conjunto.

\section{$\S 2$}

Entendemos por «conciencia moral» la capacidad discursiva del agente mediante la cual él puede integrar sus actos pasados desde una perspectiva actual, tal que ésta le permite revisar, parcial o totalmente, el sentido que esos actos tuvieron en el momento de ser llevados a cabo, a fin de incorporarlos a la unidad narrativa de su propia vida de modo consistente con su punto de vista prudencial o moral en el presente, y la capacidad argumentativa de incorporar

\footnotetext{
"Ricoeur, 1990.

4 Kohlberg, 1981.

s Habermas, tomo 2, 1981; 1988, pp. 181-247.

5 Tugendhat, 1979, pp. 245 y ss.

? Ricocur, 1990, pp. 186 y ss, y 208 y ss.
} 
en la deliberación de sus acciones futuras el punto de vista de las personas afectadas por su acción, potencialmente toda la comunidad, que se manifiesta en la certeza de que existen expectativas normativas justificadas por parte de todos los demás miembros de la sociedad de que el agente no lesionará las normas que protegen la dignidad de cllos como personas. Es mediante la actividad reflexiva sobre su pasado y deliberativa sobre su futuro que la conciencia integra discursivamente su existencia como una unidad, al menos bajo la forma de un emprendimiento permanentemente renovado. De este modo confluyen los dos ejes sobre los que fluye la vida del sujeto autónomo, la relación consigo mismo y la relación con los otros, en un núcleo que organiza las emociones y las actitudes discursivas, es decir, conectando experiencias y emociones con puntos de vista evaluativos, mediante el cjercicio de la reflexión interpretativa y de la prudencia, e interacciones, expectativas sociales y principios de la moralidad, mediante el despliegue argumentativo de la razón práctica.

Como señalé antes, Ricoeur ha caracterizado cada uno de estos ejes con una forma distinta de relación del sujeto consigo mismo: el eje a lo largo del cual éste proyecta su visión de la buena vida, que Ricoeur denomina «la perspectiva ética», corresponde a «la estima de sí»; el eje de interrelación con los otros sujetos, que él denomina la "perspectiva moral o deontológica», corresponde al «respeto de sí»: «así, estima de sí y respeto de sí representarán conjuntamente los estadios más avanzados de este crecimiento que es al mismo tiempo un despliegue de la ipseidad» ${ }^{8}$. De este modo se entrecruzan permaneritemente en la conciencia cuestiones concernientes a la vida moral, es decir, a las formas sociales de autorrealización y a los proyectos correlativos de la buena vida, por una parte, y cuestiones normativas, es decir, relacionadas de modo más estricto con cl ámbito de la moralidad y, por ende, con cl de las interacciones con los demás sujetos de la sociedad, por la otra. Unas y otras están preformadas en el mundo moral, atravesado por distintas tradiciones en convivencia frecuentemente conflictiva, en medio de las cuales el sujeto habrá de formarse a partir del peculiar nudo de influencias biológicas, psicológicas, sociales y culturales que confluyen en él a través de su mapa genético, su historia familiar, social, religiosa, etc. Es claro que la perspectiva historicista, escogida por la hermeneútica y el comunitarismo, pondrá el acento sobre las particularidades de este entrecruzamiento y hará del sujeto práctico un ser forzado a aceptarse tal como el destino lo formó o a perderse para siempre en la inautenticidad. De un modo más matizado, tanto Taylor como Ricoeur pretenden conservar lado a lado las dos perspectivas, la de la estima de sí y la del respeto de sí, dando primacía a la primera sobre la segunda: en otros términos, porque vivimos proyectándonos en un ideal de buena vida, integramos el respeto de nosotros mismos con el respeto debido a los otros ${ }^{9}$. En lo que sigue examinaré la relación entre las dos nociones centrales que aparecen aquí

Ricoeur, 1990, p. 201.

"Ricoeur, 1990 , pp. 237 y ss.; Taylor, 1989, pp. 489 y ss.; 1991 , pp. 71 y ss. 
confrontadas, la de autonomía y la de autorrealización, especialmente aquella forma de autorrealización propia de la modernidad que conocemos como «autenticidad» ${ }^{10}$.

\section{$\S 3$}

El tipo de argumentos a los que debemos apelar en discusiones como la que estoy llevando a cabo sobre la identidad del sujeto es aquel que Taylor ha caracterizado como un sustituto actual de los argumentos trascendentales descubiertos por Kant y utilizados desde entonces especialmente por la fenomenología o el último Wittgenstein ". En suma, el quid del argumento consiste en poner al descubierto aquellos supuestos conceptuales que una determinada tesis asume, de modo tal que ella se vea forzada a admitirlos como las premisas evidentes de las que, a sabiendas o no, había partido o presuponía como válidas. Por cierto, como también señala Taylor, tales argumentos suelen concluir en paradojas, precisamente porque las evidencias a las que deben apelar no siempre son las mismas para todos. A pesar de ello, es inevitable recurrir a estos argumentos, a pesar del riesgo de circularidad que conllevan, cuando lo que se pretende probar es previo a todo dato empírico, precisamente porque se refiere al marco conceptual dentro del cual habrán de ubicarse después los hechos históricos.

Antes, pues, de discutir si nos encontramos ante una irreductible oposición entre dos ideales distintos y en mutua competencia de la buena vida, el de la autonomía y el de la autenticidad, como parecen sostener en última instancia Taylor y Walzer al poner en primer lugar entre los factores constituyentes de la identidad del sujeto moral moderno su participación en una forma de vida moral densa ${ }^{12}$, es necesario hacer una distinción sobre los significados posibles del término «autonomía». A mi modo de ver, debemos distinguir entre dos significados distintos, que voy a denominar de la siguiente manera: $a$ ) «autonomía postulada», y b) «autonomía realizada».

Comenzaré por la primera, a): la autonomía postulada. Esta forma de autonomía es aquella que atribuimos a cada miembro de la sociedad y, eventualmente, a todos los miembros del género humano, cada uno de los cuales tiene interés en defenderla tanto para sí como para los otros miembros a través de la vigencia de ciertos principios universales que se comprometen a respetar. Precisamente porque es postulada, no puede ser refutada por situaciones concretas en las que de hecho un individuo particular no esté en situación de defender o cjercer esa autonomía: como por ejemplo los niños o los enfermos en estado de coma, etc. Es por ello mismo que se puede atribuir universalmente,

\footnotetext{
${ }^{10}$ Trilling, 1972, pp. 93 y ss.

"Taylor, 1995, pp. 20-33.

12 Walzer, 1994, pp. 85 y ss.
} 
como un modelo abstracto, que yo he intentado esbozar en Moralidad, bajo la forma de una concepción formal del bien, que incluye exclusivamente la capacidad de articular los propósitos de cada agente mediante la formulación de un correspondiente «silogismo práctico», En otros términos, «la condición sine qua non de la autonomía [postulada] de toda persona reside en el desarrollo de su pridencia hasta el punto de poder ponerla en práctica en el modo de proyectar, conducir y revisar su concepción de la propia vida" ${ }^{13}$.

$\mathrm{La}$ autonomía realizada, en cambio, $b$ ) es aquella que indica de modo positivo cómo es posible llevar a cabo del mejor modo posible las aspiraciones propias de todo ser racional a la felicidad y la perfecciôn, en el sentido de la plenitud de las propias capacidades intelectuales y disposiciones del carácter. Aquí están en competencia las distintas propuestas de una buena vida que se han ido desarrollando a lo largo de la historia cultural y religiosa desde la Antigüedad hasta nuestros días. Por cierto, depende de la propia orientación filosófica la elección de las formas de vida aún vigentes en la sociedad actual, especialmente luego de la desaparición de las utopias basadas en ciertas metafísicas de la historia, como el marxismo. Sin duda, podrán aparecer otras propuestas en el futuro, pero hoy en día se presentan dos ideales de la buena vida como los potenciales aglutinadores de la multiplicidad de episodios y vivencias del sujeto a fin de conferirle sentido y unidad a su existencia: i) un ideal (originalmente filosófico) de autonomía, y ii) el ideal moderno de la autenticidad.

En ambos casos - y es ésta mi tesis fuerte al respecto- está presupuesta la vigencia previa de la autonomía $a$ ), sin la cual ninguno de los dos ideales podría ser asumido por los sujetos, ya que no tendrían alternativas para su elección, como sucede en las sociedades tradicionales a raíz de la vigencia de concepciones monopólicas (religiosas, metafísicas o ideológicas) del mundo. En efecto, la autonomía a) no impone ninguna forma especial de vida, sino que se limita a excluir todos aquellos modelos que en la persecución de sus fines choquen con los principios de un ética universalista y de un sistema de derechos como el representado por la Carta de los derechos humanos. Ahora bien, no cabe duda de que tanto un ideal de autonomía b) como el ideal post-romántico de la autenticidad se han podido erigir y desarrollar únicamente sobre la base que provee la vigencia de tales principios y garantías, sin la cual tanto el uno como el otro no se hubieran podido jamás imaginar.

\section{$\S 4$}

Un examen inevitablemente somero de los rasgos más distintivos de ambos ideales de vida contrapuestos hará más claro el alcance de mi afirmación. En la reciente bibliografía se han expuesto las notas más distintivas de ambos

1.3 Guariglia, 1996, p. 197. 
modelos que es posible sintetizar en unas pocas líneas ${ }^{14}$. Comenzaré, pues, con i) el ideal de la autonomía.

i) Ha sido una de las más repetidas objeciones contra las propuestas universalistas el sostener que la propia concepción de un sujeto autónomo era una construcción o que no se sostenía en los hechos, o que, si lo hacía, era solamente como uno de los valores implícitos en una cierta concepción liberal de la vida. En efecto, se afirma, un sujeto no comprometido con sus propios fincs, es decir, escindido de las metas que le vienen impuestas por su propia comunidad, $y$, en consecuencia, privado de todos los atributos que le confieren una cierta identidad social, cultural, idiomática, religiosa y/o política, no es más que un fantasma sin carnadura que no ha existido más que en la imaginación de los pensadores de raigambre kantiana, Como señalé antes, estas objeciones caen en el vacio desde el momento en que lo que los comunitaristas caractcrizan de esta manera no es, en absoluto, el ideal específico de autonomía $b$ ), que trataremos aquí de precisar, sino solamente una abstracción teórica tanto en el plano ético como en el jurídico, a saber, la autonomía a), que, en tanto postulada, nunca puede pasar, como todo conjunto de normas, de un plexo de derechos y garantías reconocidas para cada uno de los ciudadanos de una sociedad democrática, independientemente de que la realicen o del grado de realización al que puedan alcanzar. A diferencia de ésta, la tradición liberal kantiana elaboró una cierta concepción del modo de llevar más plenamente a cabo las potencialidades intrínsecas al ser humano como sujeto de la vida moral, de modo tal que incorporara entre sus fines cicrtos ideales de perfección. Kant mismo hizo una propucsta de este tipo en la segunda parte de su Metafísica de las costumbres, en la que en cierta forma se recogen aspectos y temas de la tradición de la vida de la virtud o de la buena vida que se remontan a la Antigüedad ${ }^{15}$. En la Doctrina de la virtud, él trata específicamente de aquellos deberes en sentido amplio, es decir, que van más allá del deber estricto determinado por el derecho, y que establecen fines para las máximas del sujeto moral; por tanto, no aquellos fines que ya tenemos naturalmente a los que la ley moral restringc, sino aquellos otros que debemos tener de acuerdo con un ideal de perfección humana, para los cuales son necesarios los dos componentes clásicos de la virtud: fortaleza del carácter y sabiduría práctica ${ }^{16}$. Por cierto, esta propuesta de la buena vida, que recoge y prolonga en cierto modo la tradición filosófica aristotélica y estoica, recomienda como la mejor candidata para alcanzar del modo más pleno la propia autonomía a la vida que pone

${ }^{4}$ El presente trabajo estaba ya redactado casi en su totalidad cuando me llegó el reciente libro de C. Thiebaut, 1998, cuyo Ensayo segundo está dedicado a discutir la oposición entre alógica de la autonomia» y alógica de la autenticidad». Mi propia posición, como se verá, concuerda en lo esencial con la de Thiebaut, salvo en algunas cuestiones de detalle que lamentablemente no puedo discutir con la extensión que se merecen.

15 Para una discusión más extensa de este punto remito a Guariglia, 1999, en prensa.

to Cfr. Engstrom, 1997, pp. 23 y ss.; véanse además los trabajos contenidos on Engstrom y Whiting (comps.), 1996. 
su meta en el ejercicio de la virtud o del deber como un fin en sí mismo. Se trata, pues, de lo que J. Rawls denominaría ahora una concepción comprensiva del bien, sustentada en una cierta posición no neutral, al menos con respecto a cuáles deben ser las acciones de los hombres y las mujeres en el seno de una buena sociedad. Dicho en otros términos, la autonomía $b$ ) se propone como la mejor manera de llevar a cabo las posibilidades abiertas por la autonomía $a$ ), pero de ningún modo como la única.

ii) La segunda concepción de la buena vida a la que me voy a referir no tiene la prosapia filosófica de la anterior, sino que emerge en época más reciente, hacia fines del siglo xviI, y se desarrolla plenamente durante el período romántico en el xIx, del cual nos llega a nosotros. Taylor ha mostrado los orígenes de esta nueva noción en las disputas teológicas dentro del protestantismo inglés en torno al modo de concebir los mandatos divinos. De los platonistas de Cambridge proviene este modo de concebir el llamado de Dios como una voz interior, que indica la vía por donde realizar su camino en la tierra. Desacralizada en la teoría de los sentimientos morales de Francis Hutcheson, esta reivindicación de los propios sentimientos se extiende más allá de la esfera moral y penetra todos los órdenes de la vida interior ${ }^{17}$. La originalidad de esta concepción de la vida no se limita al campo moral; es más, ni siquiera es en este ámbito en donde encuentra un espacio propicio para expandirse ilimitadamente, sino más bien en esa nueva dimensión de la sensibilidad moderna que se desarrolla bajo la forma de relaciones sentimentales entre los sujetos, cada uno de los cuales expresa en y a través de ellas una manera única y original de vivir su vida. Por cierto, es claro que de un punto de partida como éste es imposible extraer ninguna articulación previsible y razonable de conducta, sino más bien la sola afirmación del derecho de una voluntad de libertad extrema que se plasma en el terco cultivo de la originalidad individual hasta alcanzar los extremos de lo sublime o lo grotesco ${ }^{18}$. Dos pensadores tan distantes entre sí como John Stuart Mill y Friedrich Nietzsche recogieron conceptualmente esta nueva dimensión del ser humano en la modernidad, el primero en el capítulo III de On Liberty, que se titula precisamente «Of Individuality, as One of the Elements of Well-Being»; cl segundo en diversas partes de su obra, pero especialmente en la Segunda sección de la Genealogía de la moral ${ }^{19}$. Sea a través de la sobria conciliación entre las pretensiones del individuo y de la sociedad que recomienda el primero, sea en la exarcebada afirmación del «individuo sobcrano» que propugna el segundo, es evidente en ambos casos que lo que se reivindica es el reconocimiento del desarrollo y la expansión de la individualidad en su autenticidad sin interferencias provenientes del mundo cotidiano o, para decirlo con Heidegger, de la «habladuría

${ }^{17}$ Cfr. Taylor, cap. 15,1989 , pp. 248 y ss.; 1995 , pp. 225 y ss.

${ }^{18}$ Cfr. Trilling, 1972, pp. 94 y ss.

${ }^{19}$ Este aspecto del pensamiento de Nietzsche ha sido bien expuesto por Stegmaier, 1994, pp. 136 y ss. 
de lo uno\%. Sin duda, pese a estos contactos filosóficos, el campo de la concepción de la vida auténtica es propiamente social y estético, no moral, por lo que no es sorprendente que haya encontrado sus formulaciones conceptuales más luminosas en personajes de ficción, como Lucien de Rubempré en las Ilusiones perdidas de $\mathbf{H}$. de Balzac, en el siglo pasado, o en Adrián Leverkühn del Doktor Faustus de T. Mann, en nuestro siglo ${ }^{20}$.

Taylor ha puesto en conexión este rasgo de la personalidad moderna con las exacerbadas reacciones durante las últimas décadas en las reivindicaciones culturales y religiosas, tanto de pueblos anteriormente colonizados frente a la cultura de sus ex colonizadores, como de aquellas minorías en el interior de una misma sociedad, como por ejemplo las mujeres, que no se sienten reconocidas en su propia peculiaridad y originalidad. La concepción de una vida auténtica como forma por antonomasia de autorrealización se opone, así, como una nueva concepción de la buena vida, a las previamente existentes, las que, a su modo, habian encontrado su legitimidad moral y política en el sistema normativo imperante en especial en las modernas sociedades democráticas. La irrupción de estas nuevas concepciones, difíciles de acomodar dentro de los principios de un ética universalista y de un sistema de derechos como el representado por la Carta de derechos humanos, estaria haciendo estallar el sistema de la moralidad, por así decir, desde dentro ${ }^{21}$.

${ }^{20}$ Cfr. Trilling, 1972, pp. 99-100: «Lo que la audiencia espera del artista [...] y lo que el artista piensa que debe darle resulta ser la misma cosa. Sabemos, por cierto, qué es: se trata del sentimiento de la existencia. Un sinónimo para este sentimiento de la existencia es aquella "fuerza" que, según nos dice Schiller, "el hombre trajo consigo del estado salvaje" y que el, Schiller, tiene tanta dificultad en preservar en una cultura altamente desarrollada. El sentimiento de existencia equivale a sentirse un ser fuerte. Que no significa "poderoso": Rousseau, Schiller, Wordsworth no se refieren a una energía ditigida hacia fuera, a fin de atacar y dominar el mundo, sino más bien a esa energía que se esfuerza para que el centro se mantenga firme, que la circunferencia del yo siga inquebrantable, que la persona quede integra, impenetrable, perdurable y autónoma tanto en su existencia como en su acción [...]. A través del siglo xix el arte tuvo como una de sus principales intenciones la de inducir en la audiencia el sentimiento de la existencia, la de rescatar la fuerza primitiva que una cultura altamente desarrollada habia disminuido [...]. Con el avance del siglo, el sentimiento de existencia, la sensación de ser fuertc, es subsumida panlatinamente bajo la concepción de la autenticidad personal. La obra de arte es, ella misma, auténtica en razón de su propia autodefinición: se la entiende como algo que existe por las leyes de su propia existencia, que incluye el derecho de incorporar asuntos penosos, innobles o socialmente inaceptables. De modo semejante, el artista busca su autenticidad personal en su completa autonomía: su meta es la de definirse a sí mismo de un modo tan completo como la obra de arte que él crea. Para la atidiencia, su expectativa es la de que a través de la comunicación con la obra de arte, que puede ser reluctante, desagradable y aun hostil, ella adquiera la autenticidad de la cual el objeto [de arte] mismo es el modelo y el artista el ejemplo personal [...]. La auténtica obra de arte nos instruye acerca de nuestra inautenticidad y nos conjura para que la superemos.»

${ }^{21}$ Cfr. Taylor, 1995, pp. 225-226. 


\section{$\$ 5$}

Si retomamos la tesis que sostuve en $\S 1$, a saber, que solamente a partir de la prioridad que concedamos a la autonomía sobre cualquier otra concepción de la buena vida es posible explicar la unidad o identidad propia del sujeto moral a través de las múltiples vicisitudes que atraviesa en su vida, de modo tal que la identidad, en el sentido de ipseidad del sujeto moral, proviene únicamente de la unidad narrativa de una vida, cementada por su conciencia moral, entonces podemos aplicar este criterio de identidad a los dos postulados antes expuestos. Ahora es posible reformular la tesis original de la siguiente manera: tanto el ideal de autonomía $b$ ), constituido por un proyecto de vida centrado en la búsqueda del desarrollo armónico de nuestras propias capacidades morales, cognitivas y emocionales, como el ideal de autenticidad, centrado en el ansia insaciable de encontrar una individualidad que no se desintegre en «lo idéntico», para utilizar la terminología del último Adorno, son, prima facie, formas de autorrealización compatibles con la autonomía $a$ ), cuya vigencia ambos ideales presuponen. Por cierto, la condición prima facie que establezco tiene dos aspectos distintos que es conveniente destacar. En primer lugar, como señalé antes, la autonomía a) contiene un concepto difuso o indeterminado de la subjetividad, en el que solamente se estipula aquello a lo que ésta tiene derecho a aspirar en las condiciones de una sociedad moderna y democrática. Desde ese punto de vista, es una concepción general de la autonomía, que cesa tan pronto precisamos sus diferentes y múltiples particularidades ${ }^{22}$. Solamente se mantiene como una unidad jurídica $\mathrm{y}$, por lo tanto, hipotética, dependiente de un cierto orden constitucional democrático, que garantiza esos derechos para las personas sujetas a ese orden, y, más allá de los límites de una constitución nacional, en el orden jurídico internacional, que establece la validez y exhorta a la vigencia de ciertos principios éticos universales, los derechos humanos. En segundo lugar, los derechos personales y las capacidades morales comparten un rasgo básico de las propiedades disposicionales, en el sentido de que todas ellas no son propiedades que permanecen siempre en un mismo estado, sino que se actualizan, dadas determinadas condiciones. Así, podemos entender el color de una cosa, por ejemplo una manzana roja, como la disposición de esa manzana, basada de alguna manera en sus propiedades físicas, de causar en el observador humano normal, bajo condiciones normales, una experiencia visual correspondiente, en estc caso, al rojo. De un modo similar, podemos entender los derechos y las virtudes como la disposición a llevar a cabo detcrminados comportamientos, previstos como legal y/o éticamente válidos y sustancialmente posibles, dados determinados

${ }^{2 z}$ A mi modo de ver, esta oposición entre la «autonomía general» y las «autonomías particulares" recoge la fructifera distinción entre la «voluntad general» y la "voluntad particular» introducida por Hegel en $R P h, \$ 21$ y ss. 
estímulos y oportunidades sociales. De este modo, se hace evidente que las formas de autorrealización que aspiran a coronarse como los modelos posibles de una buena vida para el sujeto moderno están todas ubicadas en una escala gradual y comparativa de posibilidades de realización, que es imposible agotar de un modo conceptual a priori.

Con esta última conclusión puedo dar por probada mi tesis original, a saber, que solamente a partir de la prioridad que concedamos a la autonomía sobre cualquier otra concepción de la buena vida, es posible explicar la unidad e identidad propia del sujeto moral moderno a través de las múltiples vicisitudes que atraviesa en su vida. Es ahora claro que la autonomía de la cual se trata es la que he denominado autonomía $a$ ), cuyas características he discutido en el párrafo anterior. Por cierto, como intenté mostrar en los dos últimos capítulos de Moralidad, la formación del sujeto moral consiste precisamente en la admisión progresiva de su identidad como sujeto autónomo, dueño de una conciencia moral y digno de reconocimiento, en el medio de una sociedad de otros sujetos morales con los mismos atributos. Sin este umbral, no hay base alguna sobre la cual el sujeto pueda construir su propia identidad, si bien esa base no le garantiza que efectivamente consiga integrarla a lo largo de una vida. Es aquí donde los distintos idcales de vida entran en competencia: asumir uno de ellos es siempre riesgoso y a veces hasta destructivo de la propia autonomia ${ }^{23}$. Si éste es el riesgo que se corre, entonces, a la inversa, lo que se arriesga es precisamente la unidad e identidad de la continuidad narrativa de la propia vida, que no es simplemente un depósito desordenado de episodios desconectados e incoherentes entre sí, sino que adquiere continuidad y consistencia merced al sentido y a la articulación que le confiere a esa multiplicidad de experiencias la conciencia reflexiva autónoma. Sobre este aspecto, sin embargo, no hay límites fijos: no podemos decir «aquí termina la conciencia de un sujeto autónomo» o «esto ya no tiene más ningún sentido». Por otra parte, también es innegable que, como señala Trilling con acierto (ver nota 20), la obra $y$ la vida de determinados artistas, como un P. Picasso o un S. Becket, son modelos al mismo tiempo de autenticidad en la labor estética y de autonomía y consistencia en la vida del creador. Dicho de otro modo, no hay prescripciones exitosas que garanticen el logro definitivo de la identidad a todo sujeto moral moderno, sino que ésta está, de igual modo que la autonomía, en permanente ricsgo de perderse, como, por lo demás, ocurre a diario en nuestras sociedades golpeadas por epidemias tanto psíquicas como sociales de marginados, tóxico-dependientes y border-lines.

Con esto último no niego la dimensión social que ha tenido y tiene el concepto de «identidad del sujeto», sobre lo que han insistido particularmente

${ }^{23}$ Quizá no exista ejemplo más ilustre y al mismo tiempo más penoso de esta experiencia de una «decision» en contra de la propia autonomía que aquella a la cual, en nombre de la autenticidad del pueblo alemán, Heidegger exhorta a sus colegas y conciudadanos a tomar a favor de Hitler en el plebiscito de noviembre de 1933; cfr. Farías, 1989 , pp. 220 y ss. 
los comunitaristas. Se trata sólo de considerar la cuestión desde una perspectiva distinta, a saber; desde la altura normativa provista por una ética universalista munida del respaldo que proporciona la Declaración de los derechos humanos, como el sustento más universal y firme que la humanidad ha alcanzado para construir y desarrollar sociedades justas y democráticas. Ahora bien, no es posible negar que la libertad propia que estos mismos derechos conceden son una pesada carga para el individuo aislado, arrojado a una sociedad cada vez menos solidaria y cada vez más colonizada por las relaciones de mercado. En estas condiciones, la tentación será grande por adoptar una falsa identidad, aferrándose a cosmovisiones metafísicas o religiosas del mundo, provenientes de tradiciones que se remontan a las sociedades jerárquicas constituidas hacia el final de la era antigua, las que asignan a cada sujeto un lugar en el mundo. Es éste, a mi juicio, el secreto vínculo que une a ciertas visiones tradicionalistas con sus correlatos desacralizados del comunitarismo. Al contrario, una posición universalista asume $\sin$ atenuantes las condiciones del mundo postmetafísico de hoy, sacudido por la más profunda transformación tecnológica y económica de la historia. En estas condiciones, el sujeto moderno está inevitablemente forzado a ser autónomo, aun cuando no lo quiera y pretenda escaparse de esa condición. Creo que el mensaje que el universalismo pretende transmitir es que el sujeto, ocultándose esta condición suya en la sociedad actual y no asumiendo los riesgos de la elección, la reflexión y la madurez, corre un peligro mucho mayor de perder su identidad sin posibilidad de recuperarla.

\section{BIBLIOGRAFIA Y REFERENCIAS}

Engstrom, S., y Whiting, J. (compiladores) (1996): Aristotle, Kant, and the Stoics, Cambridge, Cambridge UP.

Engstrom, S. (1997): «Kant's Conception of Practical Wisdom», Kant-Studien, 88 (1997), pp. 16-43.

Farfas, V. (1989): Heidegger und der Nationalsozialismus, Frankfurt, S. Fischer.

GUARIGLIA, O. (1995): Universalismus und Neuaristotelismus in der zeitgenössischen Ethik, Hildesheim-Zürich-New York, Georg Olms.

- (1996): Moralidad: Etica universalista y sujeto moral, Buenos Aires-México, Fondo de Cultura Económica.

- (1997): La Ética en Aristóteles o la moral de la virtud, Buenos Aires, Eudeba.

- (1999): "Virtud, perfección y fin último en la Metafisica de las costumbres», Revista Latinoamericana de Filosofia, 25, núm. 1 (otoño 1999), en prensa.

Habermas, J. (1981): Theorie des kommunikativen Handelns, 2 tomos, Frankfurt, Suhrkamp.

- (1983): Moralbewusstsein und kommunikatives Handeln, Frankfurt, Suhrkamp.

- (1985): Der philosophische Diskurs der Modeme, Frankfurt, Suhrkamp.

- (1988): Nachmetaphysisches Denken, Frankfurt, Suhrkamp.

- (1992): Faktizität und Geltung, Frankfurt, Suhrkamp.

- (1995): «Reconciliation through the Public Use of Reason: Remarks on John Rawls's Political Liberalism", Joumal of Philosophy, 92 (marzo 1995), pp. 109-131. 
HEGEL, G. W. F. (1970): RPh, Grundlinien der Philosophie des Rechts, Werke 7, Frankfurt, Suhrkamp.

Heidegger, M. (1957): SuZ, Sein und Zeit, Tübingen, M. Niemeyer.

KaNT, I. (1963): Werke, edición de W. Weischedel, tomo IV, Darmstadt, Wissenschaftliche Buchgesellschaft.

-.- GMS, Grundlegung zur Metaphysik der Sitten, pp. 11-102.

- KpV, Kritik der praktischen Vemunft, pp, 107-308.

- MS, Die Metaphysik der Sitten, pp. 309-636.

Kohlberg, L. (1981): The Philosophy of Moral Development (Moral Stages and the Idea of Justice), San Francisco, Harper \& Row.

KrmLICKA, W. (1995): Multicultural Citizenship (A Liberal Theory of Minority Rights), Oxford, Clarendon Press.

MacInTrre, A. (1981): After Virtue, Notre Dame, University of Notre Dame Press - (1988): Whose Justice? Which Rationality?, London, Duckworth.

McCarthY, Th. (1994): «Kantian Constructivism and Reconstructivism: Rawls and Habermas in Dialogue», Ethics 105 (octubre 1994), pp. 44-63.

MiLL, J. S. (1859): «On Liberty», en Utilitarianism, On Liberty, Essay on Bentham, editados con una Introducción por Mary Warnock, Glasgow, Collins Fount Paperbacks, 1979.

Muguerza, J. (1989): «La alternativa del disenso (En torno a la fundamentación ética de los derechos humanos)», en G. Peces Barba (ed.), El fundamento de los derechos humanos, Madrid, Debate, pp. 19-56.

- (1990): Desde la perplejidad, México-Madrid-Buenos Aires, Fondo de Cultura Económica.

Nietzsche, F. (1966): Werke in drei Bänden, editado por K. Schlechta, München, C. Hanser.

- (1886): Jenseits von Gut und Böse, 1. ${ }^{\mathrm{a}}$ ed., tomo II, pp. 563-760 y 10-243.

- (1887): Zur Genealogie der Moral, 1. ${ }^{a}$ ed., tomo II, pp. 761-900.

Nino, C. (1989): Etica y derechos humanos, 2. ${ }^{a}$ ed., Buenos Aires, Astrea.

Rawls, J. (1971): A Theory of Justice, Oxford, Oxford University Press, 1972.

- (1993): Political Liberalism, New York, Columbia University Press.

- (1995); «Reply to Habermas», Journal of Philosophy 92 (march 1995), pp. 132-180.

Ricoeur, P. (1990): Soi-même comme un autre, Paris, Editions Du Seuil.

Stegmaier, W. (1994): Nietzsches «Genealogie der Moral», Darmstadt, Wissenschaftliche Buchgeselischaft.

TAYLOR, Ch. (1989): Sources of the Self: the Making of the Modem Identity, Cambridge, Mass., Harvard University Press.

- (1991): The Ethics of Atuthenticity, Cambridge, Mass., Harvard UP.

- (1995): Philosophical Arguments, Cambridge, Mass., Harvard UP.

Thiebaur, C. (1997): «Desde Kant, de nuevo hacia Aristóteles» (reseña de Guariglia, 1996), Isegoria, núm. 17 (noviembre 1997), pp. 200-204.

- (1998): Vindicación del ciudadano, Barcelona, Paidós.

Trilling, L. (1972): Sincerity and Authenticity, Cambridge, Mass., Harvard UP.

Tugendhat, E. (1979): Selbstbewusstein und Selbstbestimmung, Frankfurt M., Suhrkamp (trad. cast., Autoconciencia y autodeteminación, Madrid-México, Fondo de Cultura Económica, 1993).

Walzer, M. (1983): Spheres of Justice, 2." ed.; Oxford, B. Blackwell, 1985.

- (1990): «The Comunitarian Critique of Liberalism», Political Theory, 18 (1990), pp. 6-23. 
- (1994): Thick and Thin: Moral Argument at Home and Abroad, Notre Dame-London, University of Notre Dame Press.

Partes de este trabajo fucron leídas en la sesión especial sobre «Ethics in the Present Decade» del Twentieth World Congress of Philosophy (Boston, 10-16 de agosto de 1998), en el Cuarto Coloquio Bariloche de Filosofía (Bariloche, 25-27 de junio de 1998) y en las Primeras Jornadas Nacionales de Ética Teórica, Pública y Aplicada, organizadas por el Instituto de Filosofía de la Universidad de Buenos Aires y el Centro de Investigaciones Filosólicas (Buenos Aires, 26-27 de noviembre de 1998). Agradezco a todos los participantes en esos eventos los valiosos comentarios que me ayudaron a mejorar la formulación de mis ideas. 